\title{
A Hidden Markov Model for Urban Navigation Based on Fingerprinting and Pedestrian Dead Reckoning
}

\author{
Jochen Seitz \\ University of Erlangen-Nuremberg \\ jochen.seitz@like.eei.uni-erlangen.de
}

Jasper Jahn

Fraunhofer Institute for Integrated Circuits IIS jasper.jahn@iis.fraunhofer.de

Javier Gutiérrez Boronat

Fraunhofer Institute for Integrated Circuits IIS javier.gutierrez@iis.fraunhofer.de
Abstract - An algorithm for pedestrian navigation in indoor and urban canyon environments is presented. It considers platforms with low processing power and lowcost sensors. A combination of Wi-Fi positioning and dead reckoning, based on a Hidden Markov Model, is used. The positions of the Wi-Fi fingerprints in the database are used as hidden states. Dead reckoning is taken for state transition and a database correlation of the Wi-Fi signal strength measurements is performed in the measurement update. The dead reckoning consists of an accelerometer driven step length estimation and a magnetic field based heading calculation. Simulations and tests demonstrate that in this way ambiguities common in Wi-Fi positioning can be solved and outages can be bridged. Therefore, higher accuracy and robustness can be achieved.

Keywords: Dead Reckoning, Hidden Markov Models, Pedestrian Navigation, Positioning, Wireless LAN

\section{Introduction}

The growing market of modern smart phones equipped with satellite receivers, wireless LAN (Wi-Fiß [15]), magnetic compasses and accelerometers not only enables new types of location based services but also provides new and cheap approaches to indoor and urban canyon navigation.

Commonly the first choice for navigation is the global positioning system (GPS). However, the lack of precision and availability of GPS in urban and indoor environments is a prevalent problem. Wi-Fi modules are usually used for communication, but can also be used for positioning [12]. Because of an increasing number of public and private access points in urban environments, Wi-Fi positioning becomes more and more attractive for pedestrian navigation [13]. The accelerometers and the magnetic compass can be used for dead

\author{
Thorsten Vaupel \\ Fraunhofer Institute for Integrated Circuits IIS \\ thorsten.vaupel@iis.fraunhofer.de \\ Steffen Meyer \\ Fraunhofer Institute for Integrated Circuits IIS \\ steffen.meyer@iis.fraunhofer.de \\ Jörn Thielecke \\ University of Erlangen-Nuremberg \\ joern.thielecke@like.eei.uni-erlangen.de
}

reckoning. While dead reckoning achieves high precision within short time periods, the positioning error of Wi-Fi positioning is not increasing with time, but less short-term accurate with an absolute error in the range of several meters [13]. Therefore the sensor data fusion of dead reckoning and Wi-Fi positioning yields a synergetic effect resulting in higher robustness and precision.

In [4] and [11] particle filters are presented for the integration of Wi-Fi positioning and inertial navigation systems. Particle filters suffer from high computational costs, depending on the number of particles computed. Kalman filters and extended Kalman filters are inappropriate for the sensor data fusion, as the assumption of Gaussian distributions is in conflict with the ambiguous outputs of Wi-Fi fingerprinting algorithms. In the field of autonomous mobile robots Hidden Markov Models (HMM) for sensor fusion have been successfully deployed, e. g. in [3] and [14].

In [7] dead reckoning has been included successfully in a HMM based on Wi-Fi positioning. In this paper we extend the HMM for Wi-Fi positioning. A continuous position estimation during $\mathrm{Wi}-\mathrm{Fi}$ outages is achieved. In Section 2 the components and equations of the HMM are presented. In Section 3 we analyze the used pedestrian dead reckoning. The general performance, drawbacks and possibilities are evaluated in Section 4 by simulations. In Section 5 experimental results in typical urban indoor and outdoor environments are presented.

\section{Hidden Markov Model}

A Markov model is called hidden if it contains an underlying stochastic process that is not directly observable, but can be observed through another stochastic process [8]. The proposed HMM is based on the discrete positions of the Wi-Fi fingerprints as the hidden states and the received signal strength (RSS) Wi-Fi measurements as the observable states. 
The iterative estimation process with the HMM is presented in Fig. 1. First an initial probability distribu-

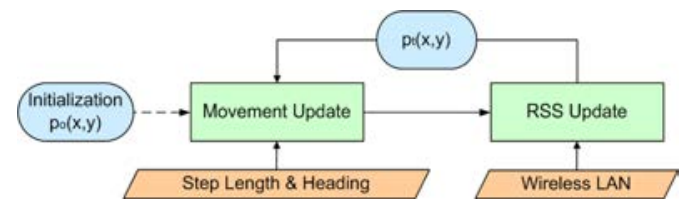

Figure 1: Iterative position estimation process

tion $p_{0}$ for the user position has to be provided. If there is no initial information, this can be assumed as an uniform distribution. In the movement update the state transition, the change of position, is based on pedestrian dead reckoning. For pedestrian dead reckoning we use the combination of traveled distance (step length), and heading angle (compass heading). For the heading we assume that it is identical to the orientation of the mobile device. We estimate the step length from the acceleration measurements using an algorithm for pedometers from [5] and the enhancement of [6]. In the RSS update a database correlation is performed to match the current RSS sensor measurements with the stored RSS values in the fingerprint database [12].

\subsection{Movement Update}

In the movement update we want to calculate the probability distribution $p\left(L_{t} \mid \mathcal{M}_{t}, \mathcal{R}_{t-1}\right)$. Assume we have already calculated the probability distribution $p\left(L_{t-1} \mid \mathcal{M}_{t-1}, \mathcal{R}_{t-1}\right)$ for the location $L_{t-1}$ at time $t-1$ given all measurements up to and including time $t-1$. $\mathcal{M}_{t-1}$ denotes the set of movement measurements and $\mathcal{R}_{t-1}$ the set of RSS measurements. The movement update is based on the Markov assumption that the user position $L_{t}=l$ at time $t$ depends only on the previous position $L_{t-1}=l^{\prime}$ and the distance and angle measurements $D_{t}$ and $\Phi_{t}$ :

$$
\begin{aligned}
p\left(L_{t} \mid \mathcal{M}_{t}, \mathcal{R}_{t-1}\right)= & \sum_{l^{\prime}} p\left(L_{t} \mid L_{t-1}=l^{\prime}, D_{t}, \Phi_{t}\right) \\
& \cdot p\left(L_{t-1}=l^{\prime} \mid \mathcal{M}_{t-1}, \mathcal{R}_{t-1}\right)
\end{aligned}
$$

The movement update for the user positions $L_{t}$ is the sum over all probabilities of previous positions $L_{t-1}$ and weighted by the transition probability $p\left(L_{t} \mid L_{t-1}=l^{\prime}, D_{t}, \Phi_{t}\right)$. Given the distance and angle measurements it can be written using Bayes law:

$$
\begin{aligned}
& p\left(L_{t} \mid L_{t-1}, D_{t}, \Phi_{t}\right)= \\
& \frac{p\left(D_{t}, \Phi_{t} \mid L_{t}, L_{t-1}\right) \cdot p\left(L_{t} \mid L_{t-1}\right)}{p\left(D_{t}, \Phi_{t} \mid L_{t-1}\right)}
\end{aligned}
$$

Assuming the distance and angle measurements are always independent of each other, we obtain:

$$
\begin{aligned}
& p\left(L_{t} \mid L_{t-1}, D_{t}, \Phi_{t}\right)= \\
& \quad \frac{p\left(D_{t} \mid L_{t}, L_{t-1}\right) \cdot p\left(\Phi_{t} \mid L_{t}, L_{t-1}\right) \cdot p\left(L_{t} \mid L_{t-1}\right)}{p\left(D_{t}\right) \cdot p\left(\Phi_{t}\right)}
\end{aligned}
$$

In case of the transition probability $p\left(L_{t} \mid L_{t-1}\right)$, we could take into account, e. g., that a movement to neighboring locations is more likely than to very distant locations. However, using the movement measurements $D_{t}$ and $\Phi_{t}$ we have already a reliable measure for what locations are more likely than others. For simplicity we assume that $p\left(L_{t} \mid L_{t-1}\right)$ is a constant for all pairs $\left(l, l^{\prime}\right)$. Inserting now Eq. (3) into Eq. (1) we finally obtain for the movement update:

$$
\begin{aligned}
& p\left(L_{t} \mid \mathcal{M}_{t}, \mathcal{R}_{t-1}\right)=c \cdot \sum_{l^{\prime}} p\left(D_{t} \mid L_{t}, L_{t-1}\right) \\
& \cdot p\left(\Phi_{t} \mid L_{t}, L_{t-1}\right) \cdot p\left(L_{t-1} \mid \mathcal{M}_{t-1}, \mathcal{R}_{t-1}\right)
\end{aligned}
$$

Here we have collected all probabilities independent of $l^{\prime}$ in the constant c. This constant can be determined at the end of the movement update by normalization, since the calculated distribution is a probability distribution, summing over all possible locations $L_{t}=l$ must yield 1.

In Eq. (4) the probability distributions characterizing the distance and angle measurements $p\left(D_{t} \mid L_{t}, L_{t-1}\right)$ and $p\left(\Phi_{t} \mid L_{t}, L_{t-1}\right)$ are assumed to be Gaussian:

$$
\begin{gathered}
p\left(D_{t}=d_{t} \mid L_{t}=l, L_{t-1}=l^{\prime}\right)= \\
\frac{1}{\sqrt{2 \pi} \cdot \sigma_{d}} \cdot e^{-\frac{1}{2 \sigma_{d}^{2}}\left(d_{t}-d\left(l, l^{\prime}\right)\right)^{2}} \\
p\left(\Phi_{t}=\varphi_{t} \mid L_{t}=l, L_{t-1}=l^{\prime}\right)= \\
\frac{1}{\sqrt{2 \pi} \cdot \sigma_{\varphi}} \cdot e^{-\frac{1}{2 \sigma_{\varphi}^{2}}\left(\varphi_{t}-\varphi\left(l, l^{\prime}\right)\right)^{2}}
\end{gathered}
$$

Implicitly, we have assumed here that all measurements are taken just at the moment when the new reference point $l$ is reached. Fig. $2 \mathrm{a}$ and $2 \mathrm{~b}$ visualize these conditional probabilities for the case that the previous position $l^{\prime}$ is at the center of the coordinate system. Multiplying both conditional probabilites yields the probability distribution of Fig. 2c. Obviously, combining the distance and angle measurement gives a rather precise indication whereto the position changes.

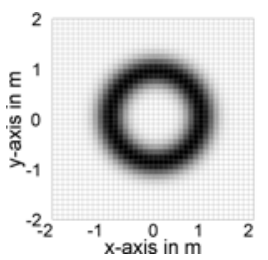

(a)

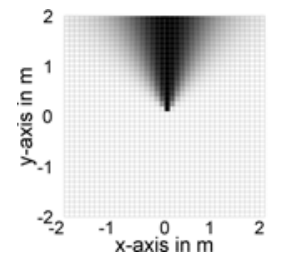

(b)

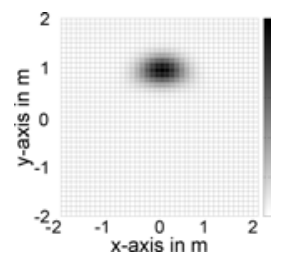

(c)
Figure 2: Transition probability distributions in gray scale, white equates probability density 0 , for (a) step length, (b) compass-heading and (c) the combination of both, with one estimated user position $l^{\prime}$ in the center 


\subsection{RSS Update}

In the RSS update we calculate the probability distribution for the location $L_{t}$ given all movements and RSS measurements up to and including time $t$ by weighting the probability $p\left(\mathbf{r}_{\mathbf{t}} \mid L_{t}\right)$ of the collected RSS measurement vector $\mathbf{r}_{\mathbf{t}}$ by the probability of the movement update from (1):

$$
p\left(L_{t} \mid \mathcal{M}_{t}, \mathcal{R}_{t}\right)=p\left(\mathbf{r}_{\mathbf{t}} \mid L_{t}\right) \cdot p\left(L_{t} \mid \mathcal{M}_{t}, \mathcal{R}_{t-1}\right)
$$

For $p\left(\mathbf{r}_{\mathbf{t}} \mid L_{t}\right)$ we use the probabilistic database correlation of [9]. A Gaussian distribution is assumed for each RSS measurement $r_{t, i}$ of access point $i$ at a fingerprint location. So a standard deviation $\sigma_{i}$ for each RSS measurement has to be stored additionally in the database. To evaluate the actual RSS measurements $r_{t, i}$ we use a probability density function with the database entry $r_{i}$ as mean and standard deviation $\sigma_{i}$. As typically Wi-Fi devices provide discrete RSS values quantized within an interval of approximately $1 d B$, we integrate the Gaussian probability distribution between $a=r_{t, i}-0.5 d B$ and $b=r_{t, i}+0.5 d B$. We repeat this procedure for all observed access points and multiply the results to obtain the probability for the measurement vector $\mathbf{r}_{\mathbf{t}}$ at location $L_{t}=l$ :

$$
p\left(\mathbf{r}_{\mathbf{t}} \mid L_{t}=l\right)=\prod_{i} \frac{1}{\sqrt{2 \pi} \cdot \sigma_{i}} \cdot \int_{a}^{b} e^{-\frac{1}{2 \sigma_{i}^{2}}\left(r-r_{i}(l)\right)^{2}} d r
$$

After the RSS update has been performed the resulting probabilities of the user position have to be normalized. The final user position $[x, y]$ can be estimated by calculating the weighted mean of all possible positions $l$, for example weighted by their probabilities $p\left(L_{t}=l \mid \mathcal{M}_{t}, \mathcal{R}_{t}\right)$ from Eq. (7). The advantage of the proposed HMM is that the database correlation is directly included in the fusion process. Ambiguities in the user position caused by similar fingerprints can be handled in the RSS update.

\section{Pedestrian Dead Reckoning}

In Section 2 the iterative position estimation process is explained assuming that after each step Wi-Fi RSS measurements are available, so that a RSS update can be performed. To enable the position estimation if not enough valid RSS measurements are present, we extend the iterative process as depicted in Fig. 3. The movement update is repeated with new step length and compass heading data as long as no valid RSS measurements are available. Reasons for these RSS interruptions or outages are typically: not enough access points in range, the Wi-Fi card of the mobile device is busy, or less than three access points answer the request for some reason - to get meaningful results from the database correlation at least three access points must have answered a request [13]. If there is no RSS update only the resulting probability distribution from

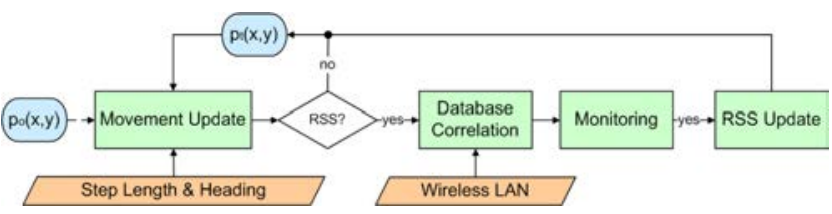

Figure 3: Extended iterative position estimation process to deal with Wi-Fi outages and to monitor the results of the RSS Update

each movement update is used to calculate the user position.

On the one hand the positioning error of Wi-Fi fingerprinting is bounded and depends on the amount and arrangement of the access points as well as the shadowing effects of the environment as e.g. examined in [9] and [13]. On the other hand the positioning error using dead reckoning is unbounded with time, but for a shorttime lower than using Wi-Fi fingerprinting. For this reason, we monitor the results from the database correlation. If the resulting probability distribution from the database correlation is highly ambiguous or does not match with the distribution from the movement update we discard the results and proceed with the next movement update. Reasons for a bad match can be e.g. measurement errors of the Wi-Fi device or local changes of the environment.

To enable the monitoring it is important to estimate the positioning error of dead reckoning if repeatedly no RSS updates have been performed. For one step the positioning error follows a Gaussian distribution as examined in [10]. The step length error $\Delta l$ is distributed with $\mathcal{N}\left(0, \sigma_{l}{ }^{2}\right)$ and the angular error $\Delta \varphi$ with $\mathcal{N}\left(0, \sigma_{\varphi}{ }^{2}\right)$. An example is depicted in Fig. 4. One step has been made

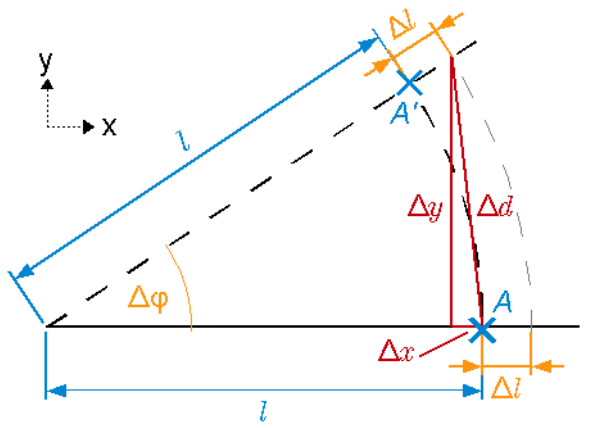

Figure 4: Positioning errors for one step in $\mathrm{x}$-direction

in x-direction with length $l$. The step length error is $\Delta l$ and the compass error is $\Delta \varphi$. Hence, the resulting position using dead reckoning is $A^{\prime}$ instead of $A$. The positioning errors $\Delta x$ and $\Delta y$ can be calculated by:

$$
\begin{aligned}
& \Delta x(l, \Delta l, \Delta \varphi)=(l+\Delta l) \cdot \cos (\Delta \varphi)-l \\
& \Delta y(l, \Delta l, \Delta \varphi)=(l+\Delta l) \cdot \sin (\Delta \varphi)
\end{aligned}
$$

and the resulting positioning error $\Delta d$ using the law of 
cosine by:

$\Delta d(l, \Delta l, \Delta \varphi)=\sqrt{\Delta l^{2}+l \cdot(l+\Delta l) \cdot\left(2 \cdot \sin \left(\frac{\Delta \varphi}{2}\right)\right)^{2}}$

The positioning errors depend on the distance $l$ and the distance and heading errors $\Delta l$ and $\Delta \varphi$, but not on the absolute heading $\varphi$. If the user moves several steps in equally distributed directions the positioning errors annihilate each other and the variance of the estimated position grows with each step. However, for pedestrians it is more likely that there are less changes of the heading.

Next we consider walking in a corridor with a constant heading. If the compass is perfect $(\Delta \varphi=0)$ and there is a step length estimation error $\Delta l$, the positioning errors of each step also annihilate each other and the variance of the estimated position grows with each step. In contrast, if the step length estimation is perfect $(\Delta l=0)$ and there is a compass heading error $\Delta \varphi$, the positioning errors do not annihilate each other. A systematic error occurs that is maximal if there is no change in heading, as presented in [10]. An example is depicted in Fig. 5. The first step and the second step

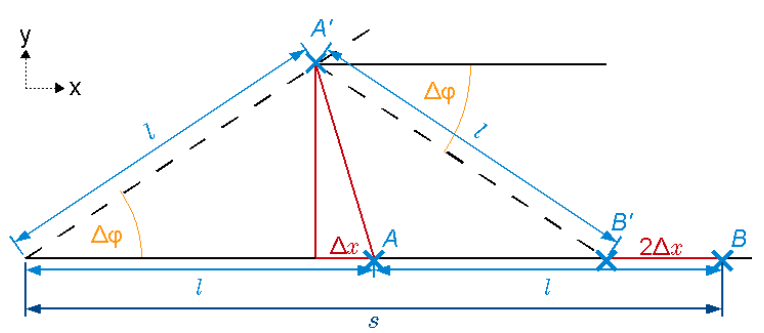

Figure 5: Positioning errors for two horizontal steps with a heading error $\Delta \varphi$ in opposite directions

with length $l$ have been perfectly estimated $(\Delta l=0)$. The compass heading errors $\Delta \varphi$ of both steps have the same amount but opposite directions. Therefore, the positioning errors in y-direction annihilate each other, but an error remains in $\mathrm{x}$-direction between the estimated and the true position $B^{\prime}$ and $B$.

This systematic error causes the estimated position to drop back from the true position. As can be seen in Eq. (9), the error grows with a larger step length $l$ and a higher compass error $\Delta \varphi$.

\section{Simulations}

To validate our models we simulated a typical Wi-Fi positioning scenario including signal shadowing. The signal strength for the fingerprinting database was calculated by modeling the path loss together with lognormal fading. Furthermore we added white Gaussian noise to an ideal step length, the compass heading and the RSS measurements.

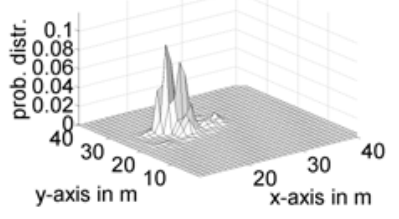

(a)

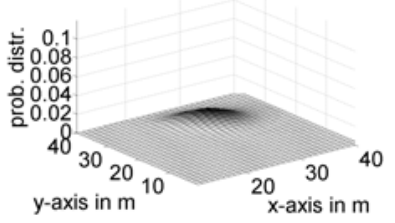

(c)

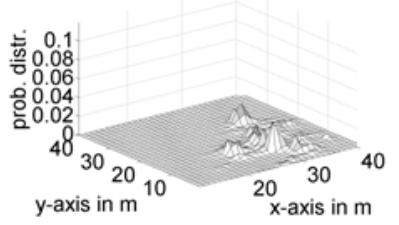

(e)

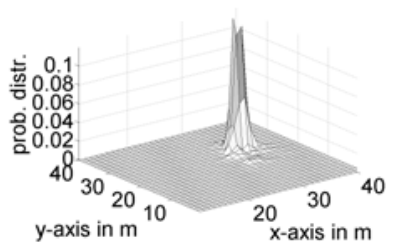

(g)

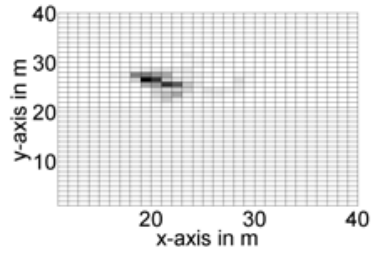

(b)

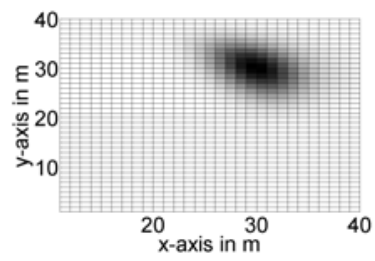

(d)

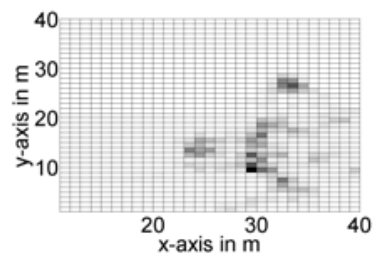

(f)

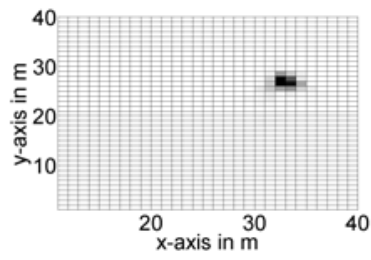

(h)
Figure 6: Steps of the iterative HMM in side (left) and top view (right): RSS update 1 (a,b), movement update with 12 steps in $\mathrm{x}$-direction $(\mathrm{c}, \mathrm{d}), \mathrm{Wi}-\mathrm{Fi}$ database correlation results $(\mathrm{e}, \mathrm{f})$ and $\mathrm{RSS}$ update $2(\mathrm{~g}, \mathrm{~h})$

Fig. 6 presents four steps of the iterative workflow. It starts with the probability distribution of the user position after a RSS update Fig. 6(a,b). Then, in the simulation, the user walks 12 steps, about $10 \mathrm{~m}$, in $\mathrm{x}$ direction. The result of the movement updates using Eq. (4) can be seen in Fig. 6(c,d). The probability distribution slides in $\mathrm{x}$-direction. Furthermore it is broadened and flattened because of step length and compass heading uncertainties. After the 12 steps new Wi-Fi measurements are available. The results of the probabilistic database correlation using Eq. (8) are presented in Fig. 6(e,f). In this case typical ambiguities appear for the user position. The correlation results are weighted according to Eq. (7) by the results of the movement update. As displayed in Fig. $6(\mathrm{~g}, \mathrm{~h})$ the ambiguities from the database correlation can be resolved.

We evaluated the benefit of including dead reckoning in the HMM compared to just Wi-Fi fingerprinting in [7]. Through simulations we investigated the possible 
improvements. By integrating the step length in the movement update the positioning error is reduced by more than $50 \%$. With the additional use of compass heading it is reduced by more than $75 \%$.

For a person walking in x-direction an example of using the step length in the movement update is shown in Fig. 7. After the first step in Fig. 7a the typical Gaussian ring is visible. After several steps the probability distribution resembles more and more a Gaussian distribution. The mean remains in the center, where the dead reckoning process started. The standard deviation increases with each step. The positioning error in $\mathrm{x}$-direction is the same as the traveled distance.

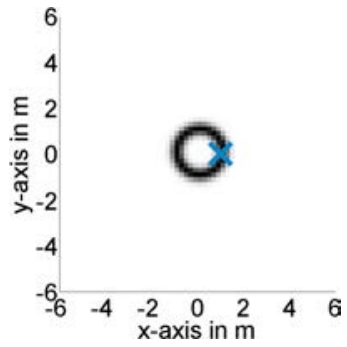

(a)

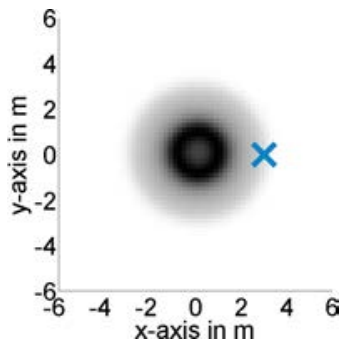

(c)

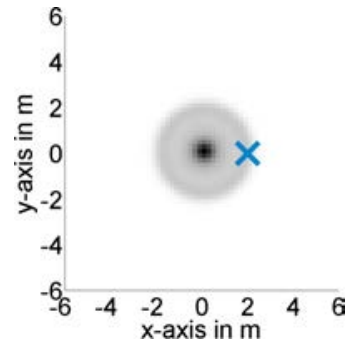

(b)

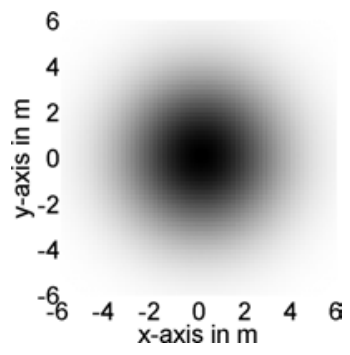

(d)
Figure 7: Probability distribution and the true position (cross) using only the step length in the movement update after (a) one, (b) two, (c) three and (d) 10 steps (true position outside view)

When additionally using the compass heading for dead reckoning the resulting distribution follows the user position, but there exists a systematic horizontal positioning error as investigated in Chapter 3. An example with a standard deviation of $45^{\circ}$ is depicted in Fig. 8. With an increasing number of steps the resulting probability distribution calculated by the movement update drops back. After 50 steps in Fig. 8d the error in $\mathrm{x}$ is about half of the traveled distance.

In Fig. 9 the relation between the standard deviation of the compass heading and the positioning error can be seen for a user walking 20 steps in the same direction. The position has been estimated using only the movement update. The simulations were performed 50 times at each different compass standard deviation to yield an averaged positioning error. The positioning error orthogonal to the walking direction (dashed line) is

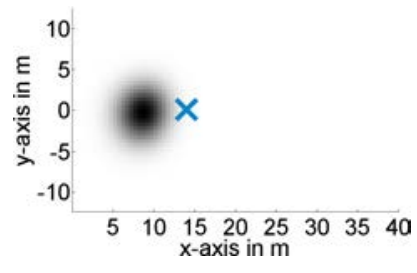

(a)

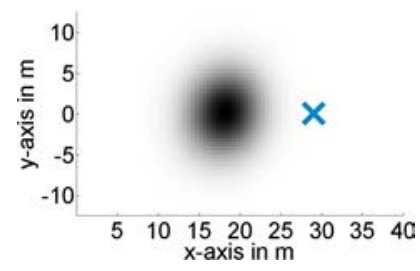

(c)

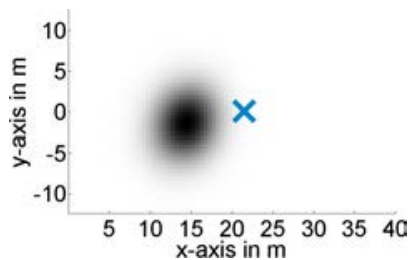

(b)

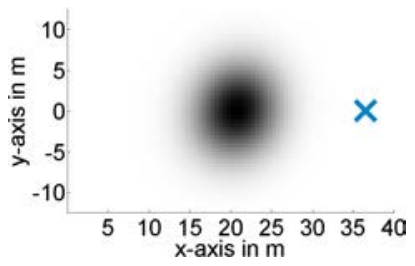

(d)
Figure 8: Probability distribution using step length and compass (with $\sigma_{\varphi}=45^{\circ}$ ) in the movement update after (a) 20 steps, (b) 30 steps, (c) 40 steps and (d) 50 steps, and the true position (cross)

in the range of meters and distributed around zero. The positioning error in the walking direction shows the systematic error investigated in Chapter 3. For standard

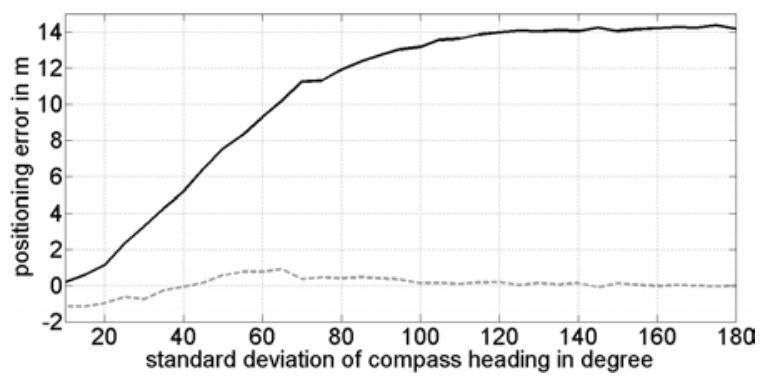

Figure 9: Average systematic positioning errors for various standard deviations for the compass heading for (a) the error orthogonal to (dashed line) and (b) in direction of the constant user heading (solid line)

deviations larger than $120^{\circ}$ the error stays on the same level of about $14.2 \mathrm{~m}$, which is close to the total traveled distance of $15 \mathrm{~m}$. Hence, for standard deviations of higher than $120^{\circ}$ the use of the compass has no effect. For low standard deviations the systematic error increases linearly. For a standard deviation of $45^{\circ}$ the systematic error is about half of the traveled distance.

The systematic error could be mitigated increasing the estimated step length by an offset or increasing the standard deviation for the step length in the movement update. These approaches are not optimal and would only work well if the user does not change his heading frequently. If the resulting probability distribution for the user position calculated by several movement updates is too erroneous, the RSS update will fail, because there will be no match with the database correlation results. 
In summary, at the moment, using the movement update without any RSS update, the number of steps the user position can still be estimated is limited. Limiting factors are the standard deviation of the step length and especially the standard deviation of the compass, because of the systematic error investigated above.

\section{Experimental Results}

In this section we compare the results from our simulations with the results from two experimental setups. We collected acceleration, magnetic compass and Wi-Fi measurements with a HTC Hero mobile phone based on the Android ${ }^{\mathrm{TM}}$ [1] operating system. The acceleration sensors and the magnetic compass are integrated on one chip. If both are used at the same time, the maximum sampling rates are approximately $30 \mathrm{~Hz}$ for the acceleration measurements and $20 \mathrm{~Hz}$ for the compass. The sampling rate for the Wi-Fi measurements was about $5 \mathrm{~Hz}$. For position estimation we included the HMM in the navigation framework of the $\mathrm{Wi}-\mathrm{Fi}$ positioning system awiloc $^{\mathrm{TM}}[2]$ of the Fraunhofer Institute for Integrated Circuits IIS.

\subsection{Office Building}

Our first scenario is the building of the Fraunhofer IIS in Nuremberg. It is a typical office building. In this case we can use our own Wi-Fi infrastructure in combination with the signals received from access points placed in other buildings and companies located nearby. To create the fingerprinting database several persons collected measurements with the presented sensing device. During the measurements they walked on previously planned paths. We used separate measurements for position estimation. In the database the identifiers of the received access points together with their RSS measurements are stored per fingerprint.

In the RSS update we used an approximation of Eq. (8) with a fixed standard deviation for the RSS measurements similar to the one presented in [12]. This was necessary because no standard deviations for the RSS measurements were stored in the database. In Fig. 10 the positioning results and the contour of the office building are presented. The user started indoors on the first floor and walked the depicted indoor path along the corridors, down the stairs to the ground floor and out of the building. He circled the building two times in different distances to the building. Then he entered the building again and walked the same indoor path as on the first floor now on the ground floor. With the use of dead reckoning in the HMM, ambiguities can be handled. Hence, the estimated positions are mainly on the path, as can be seen in Fig. 10b. The use of the compass caused difficulties. Ferromagnetic materials in the building structure caused large magnetic disturbances and the user heading was not optimally aligned

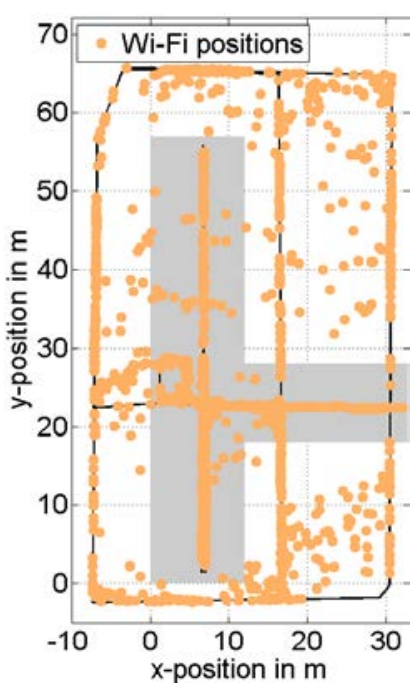

(a)

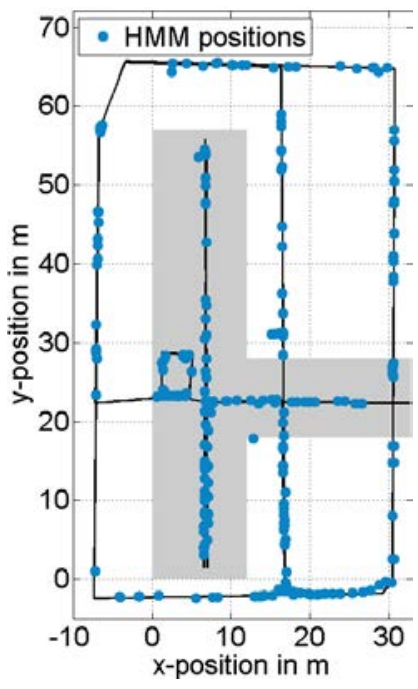

(b)
Figure 10: Reference path (solid line) within the office building (shaded areas) and around it: estimated positions of (a) Wi-Fi fingerprinting stand-alone and (b) the HMM

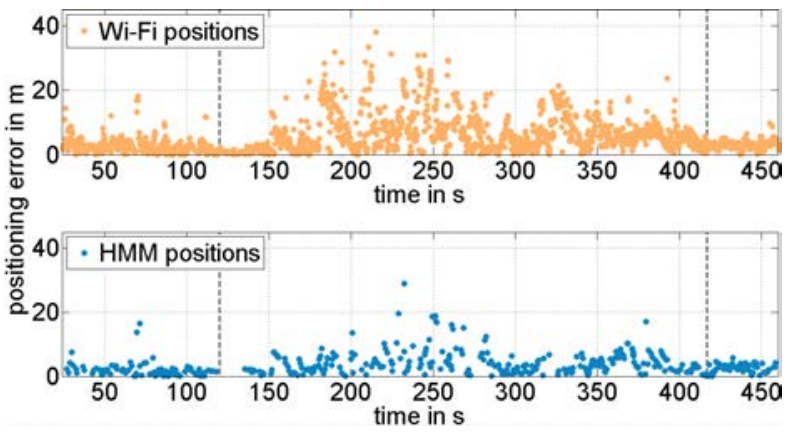

(a)

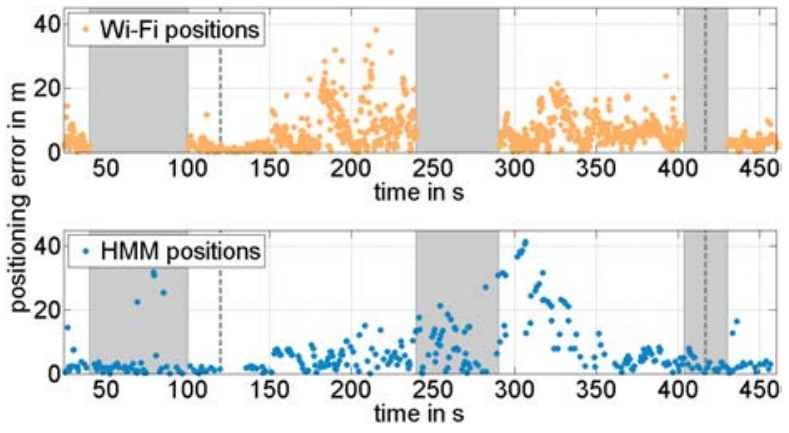

(b)

Figure 11: Positioning errors with (a) area-wide Wi-Fi coverage and (b) three outages (shaded areas) using Wi-Fi fingerprinting stand-alone and the HMM at the office building

to the orientation of the sensing device. To compensate for the not modeled misalignment we used $45^{\circ}$ as standard deviation of the compass measurements in the 
movement update.

In Fig. 11a the positioning errors for Wi-Fi fingerprinting stand-alone and using the HMM are shown. Between the dashed lines the user walked outdoors. At the beginning and towards the end the user walked indoors. The accuracy indoors is significantly higher. Using the HMM indoors and outdoors instead of Wi-Fi fingerprinting higher accuracy can be achieved, see also [7]. In and around the office building the Wi-Fi coverage is almost optimal. So there occurred no outages. To test the bridging capabilities of the proposed algorithm we removed some Wi-Fi measurements. The resulting positioning errors are depicted in Fig. 11b.

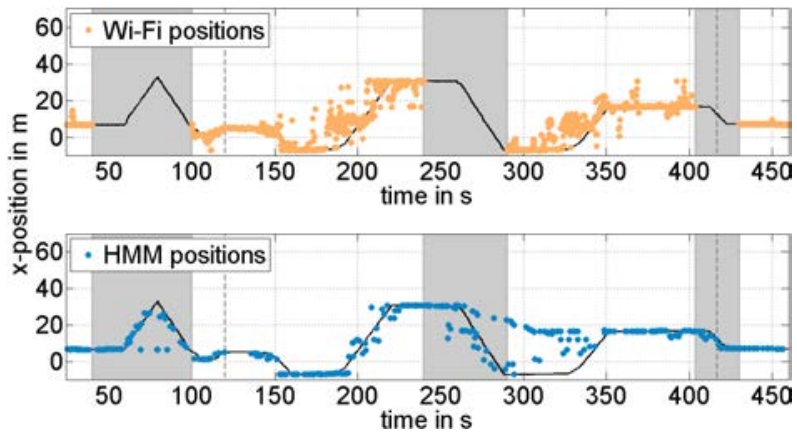

(a)

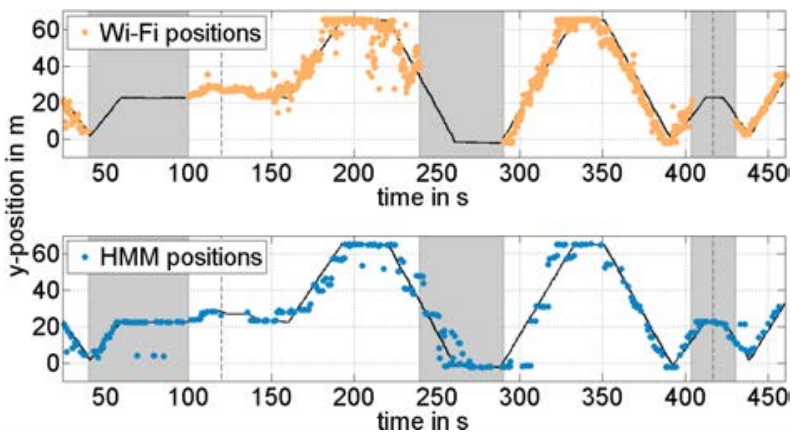

(b)

Figure 12: Reference positions (solid line) and the estimated $\mathrm{x}$ - and $\mathrm{y}$-positions with three Wi-Fi outages (shaded areas) using Wi-Fi fingerprinting stand-alone and the HMM at the office building

During the first outage, the user has been indoors, during the second outdoors and during the third he walked the first half outdoors and the second indoors. In the first and the last outage the bridging worked well, but few positions were estimated with a notable higher positioning error. This indicates that some ambiguities occurred during the estimation process with the HMM. During the outage outdoors the error grew more rapidly, but the error could be slowly reduced to normal after the outage. Fig. 12 presents the estimation results along the $\mathrm{x}$ - and $\mathrm{y}$-direction. The estimation with the HMM results indoors in probability distributions for the user position with a much smaller variance than outdoors. Because of this, higher accuracy is achieved and the HMM has to deal with less ambiguities than outdoors. Hence, the bridging of Wi-Fi outages works especially well indoors.

\subsection{Market Square}

The second scenario is the historic square in the center of Nuremberg, the famous Christmas market. The measurements were taken after the market was set-up, but before it started. No visitors were on the market. The estimation results are presented in Fig. 13 along the $\mathrm{x}$ - and $\mathrm{y}$-direction. The results using Wi-Fi fingerprinting stand-alone include four Wi-Fi outages due to worse $\mathrm{Wi}-\mathrm{Fi}$ coverage on the market square compared to the office building.

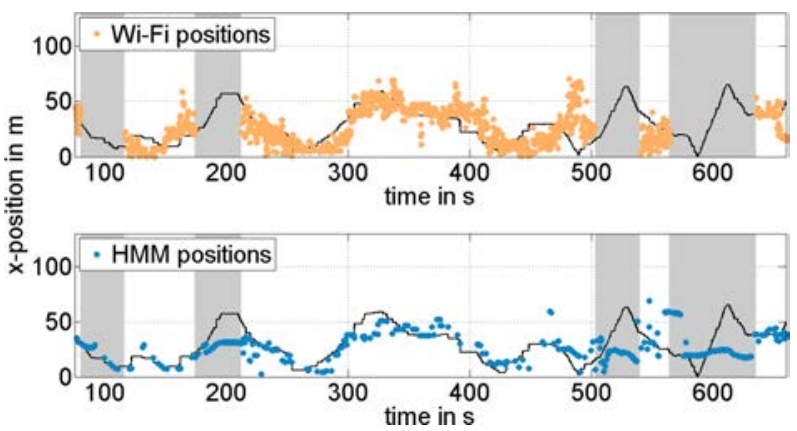

(a)

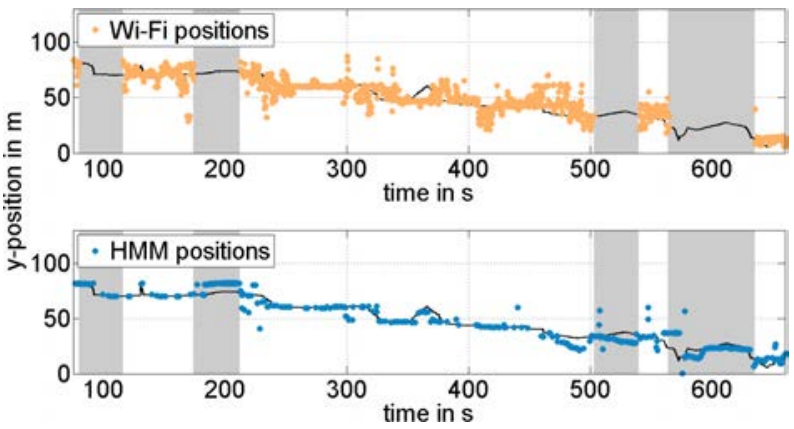

(b)

Figure 13: Reference positions (solid line) and the estimated $\mathrm{x}$ - and $\mathrm{y}$-positions with four Wi-Fi outages (shaded areas) using Wi-Fi fingerprinting stand-alone and the HMM at the market square

Because of the lack of shadowing objects, like e.g. walls in buildings, the market square is not an ideal location for Wi-Fi positioning. This causes a lot of ambiguities in the database correlation and results in large distances between two sequently estimated positions. As can be seen in Fig. 13, by using the HMM ambiguities resulting from the database correlation can be mitigated. Outages can be bridged at the expense of a degrading accuracy during outages. Furthermore, with Wi-Fi coverage the accuracy of positioning can be improved using the proposed model. 


\section{Conclusions}

A method to fuse the data of low cost sensors on mobile phones for precise and robust calculation of position and heading of pedestrians in urban environments is proposed. The presented HMM can deal with ambiguities resulting from Wi-Fi fingerprinting. The algorithm is computationally efficient because of the discrete space model, based on the positions of Wi-Fi fingerprints. Dead reckoning has been included in the movement update of the HMM. First, the benefits of the proposed algorithm and the positioning errors during Wi-Fi outages have been evaluated in simulations. The positioning errors during the outages depend significantly on the standard deviation of the compass. Second, the algorithm has been tested with real measurement data collected with commercial mobile phones in two real scenarios involving an office building and a Christmas market. Indoors $\mathrm{Wi}-\mathrm{Fi}$ positioning in general works very well, because of few ambiguities. Outdoors, positioning benefits especially from dead reckoning in the movement update of the HMM. Therefore, most ambiguities can be solved. During Wi-Fi outages the user position is calculated continuously. So higher robustness and accuracy can be achieved.

\section{Acknowledgment}

This work is funded by the Bavarian ministry of economic affairs, infrastructure, transport and technology in the scope of the strategic program "Bayern 2020". The authors want to especially thank Dipl.-Ing (FH) Stephan Haimerl of the Fraunhofer Institute for Integrated Circuits IIS in Nuremberg for his help concerning the Wi-Fi positioning system awiloc [2].

\section{References}

[1] android, "http://www.android.com", android is a registered trademark of Google Inc.

[2] awiloc, "http://www.awiloc.com", awiloc is a trademark of the Fraunhofer Institute for Integrated Circuits IIS, Germany, 2010

[3] Dieter Fox, Markov Localization: A Probabilistic Framework for Mobile Robot Localization and Navigation, Institute of Computer Science III., University of Bonn, Germany 1998

[4] H. Wang, A. Szabo, and J. Bamberger, "Performance Comparison of Nonlinear Filters for Indoor WLAN Positioning", The 11th International Conference on Information Fusion, Vol. 1, 2008

[5] Harvey Weinberg, "Using the ADXL202 in pedometer and personal navigation applications", Application Note AN-602, Analog Devices, 2002
[6] Jim Scarlett, "Enhancing the performance of pedometers using a single accelerometer", Application Note AN-900, Analog Devices, 2007

[7] J. Seitz, T. Vaupel, S. Meyer, J. Gutiérrez Boronat, and J. Thielecke, "A Hidden Markov Model for Pedestrian Navigation", Workshop on Positioning, Navigation and Communication (WPNC), Dresden, Germany, 2010

[8] L. Rabiner, and B. Juang, "An Introduction to Hidden Markov Models", ASSP Magazine, IEEE, Vol. 3, pp. 4-16, Jan. 1986

[9] Michael Wallbaum, Indoor Geolocation Using Wireless Local Area Networks, Shaker Verlag, Chair of Computer Science 4, Department of Computer Science, RWTH Aachen University, 2005

[10] O. Mezentsev, G. Lachapelle, and J. Collin, "Pedestrian Dead Reckoning - A Solution to Navigation in GPS Signal Degraded Areas" Geomatica, Canadian Institute of Geomatics, Vol. 59, No. 2, pp. 175182,2005

[11] O. Woodman, and R. Harle, "Pedestrian Localisation for Indoor Environments", Proceedings of the 10th international conference on ubiquitous computing, ACM New York, USA, pp. 114-123, 2008

[12] P. Bahl, and V. N. Padmanabhan, "RADAR: an in-building RF-based User Location and Tracking System", INFOCOM 2000. Nineteenth Annual Joint Conference of the IEEE Computer and Communications Societies. Proceedings. IEEE, Vol. 2, p 10,2000

[13] S. Meyer, T. Vaupel, and S. Haimerl, "WI-FI Coverage and Propagation for Localization Purposes in Permanently Changing Urban Areas", IADIS International Conference Wireless Applications and Computing, 2008

[14] S. Thrun, W. Burgard, and D. Fox, Probabilistic Robotics, The MIT Press, Cambridge, Massachusetts, USA, 2005

[15] Wi-Fi, "http://www.wi-fi.org," Wi-Fi is a registered trademark of the Wi-Fi Alliance, 2003 